\title{
A Flexible AMOLED Display Driven by Organic TFTs with an Inkjetted Semiconductor Layer
}

\author{
Akihito Miyamoto $^{\dagger}$, Yuko Okumoto ${ }^{\dagger}$,Kenichi Sasai ${ }^{\dagger}$ and Kiyoyuki Morita ${ }^{\dagger}$
}

\begin{abstract}
A 4-inch 80-ppi flexible AMOLED display was fabricated using organic TFTs with an inkjetted semiconductor layer on polymer gate insulator film. To suppress variations in organic TFT (OTFT) characteristics, the process damage to polymer gate insulator was remedied by surface treatments, and organic semiconductor crystallinity was improved by controlling the film thickness, giving a threshold voltage uniformity of $-0.2 \pm 0.45 \mathrm{~V}$. We also observed the non-degradation of the OTFT characteristics even after bending it 200 times over a 5 -mm radius.
\end{abstract}

Keywords: organic TFT, inkjet, threshold voltage, uniformity, flexible OLED display

\section{Introduction}

Organic thin film transistors (OTFTs) are attracting growing attention as TFTs for flexible display backplanes, especially as the manufacture of plastic substrates has the advantage of requiring a lowtemperature process. Several groups have demonstrated flexible organic light-emitting-diode (OLED) displays driven by OTFTs using organic semiconductors (OSCs) formed by thermal evaporation ${ }^{1)-4}$. However, evaporation processes are unsuitable for large-area, low-cost fabrication displays, making a printing process necessary. An inkjet-printing process has potential as a non-contact process that provides high resolution. Active-matrix organic light-emitting diode (AMOLED) displays with OSCs patterned by inkjet printing have been reported by C. Harada et al. 5) 6) and Y. Okumoto et al. ${ }^{7}$. However, an inorganic gate insulator, such as silicon dioxide, on a glass substrate is employed in their displays, so it requires a process temperature over $300{ }^{\circ} \mathrm{C}$, and the resultant display is rigid.

In this study, we developed OTFTs with a polymer gate insulator (GI) on a plastic substrate, with the OSC was fabricated by inkjet printing. We found that the threshold voltage $(\mathrm{Vt})$ of the OTFT with the polymer GI varies widely in response to the surface condition of the polymer GI and the OSC film thickness. We thus tried to minimize variations in Vt by applying surface treatments described in a previous study ${ }^{7)}$ and adjusting

Received March 2, 2014; Revised July 20, 2014; Accept July 25, 2014

$†$ Panasonic Corporation

(3-1-1 Yagumo-nakamachi, Moriguchi City, Osaka 570-8501, Japan) the OSC film thickness. We also fabricated a 4-inch 80ppi flexible AMOLED display driven by OTFTs that showed good mechanical reliability and smoothly moving images at a frame rate of $60 \mathrm{~Hz}$.

\section{Structure and Process Flow}

Fig.1 shows a schematic cross-section of a pixel in the display. A molybdenum (Mo) gate electrode was wired on a plastic substrate with a barrier layer. A $360 \mathrm{~nm}$-thick polymer film (negative-type photopolymer material), to act as the gate insulator, was fabricated using a spincoater. Mo was used for the source/drain (SD) electrodes and patterned using a wet etching process. A hydrophobic bank, made of a photosensitive polymer, was formed by spin-coating. The bank was employed as the frame for the OSC ink (a benzoporphyrin derivative), which was then patterned using inkjet printing under an $\mathrm{N}_{2}$ atmosphere. An over-coating layer and a planarization layer consisting of photosensitive polymers were then formed by spin-coating. These polymer insulators and the OSC ink were baked at $210^{\circ} \mathrm{C}$. The OLED layers

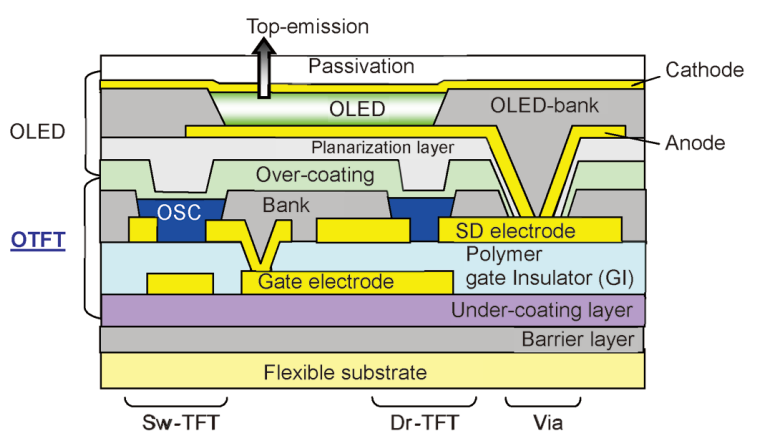

Fig.1 Schematic cross section view of a sub-pixel in a flexible OLED display driven by OTFTs. 
were fabricated by thermal evaporation, giving a topemitting-type structure.

\section{Experimental Results and Discussion}

\subsection{Improvement of Vt variability}

Vt variations in OTFTs should be kept within $1 \mathrm{~V}$ to provide high image quality in an AMOLED display. However, our experimental results showed the Vt variations in the OTFTs without any surface treatment of the polymer GI to have a wide range of $1.0 \pm 4.5 \mathrm{~V}$, as shown in Fig.2 (i). Controlling the chemical species with various self-assembled monolayers (SAMs) on the GI surface is the most commonly-adopted method of altering the characteristics of the OSC/GI interface to improve the $\mathrm{Vt}^{8)-13)}$. According to a previous study ${ }^{7}$, changes in Vt shift are attributable to residual materials such as MoOx (molybdenum oxides) that remain attached to the GI $\left(\mathrm{SiO}_{2}\right)$ surface during the deviceforming process, which may cause positive charge generation at the interface between the OSC and residual materials. Our strategy for reducing the Vt variations involved two surface treatments on the polymer GI and control of the OSC crystallinity by adjusting the film thickness.

We first tried to eliminate the $\mathrm{MoOx}$ residue on the

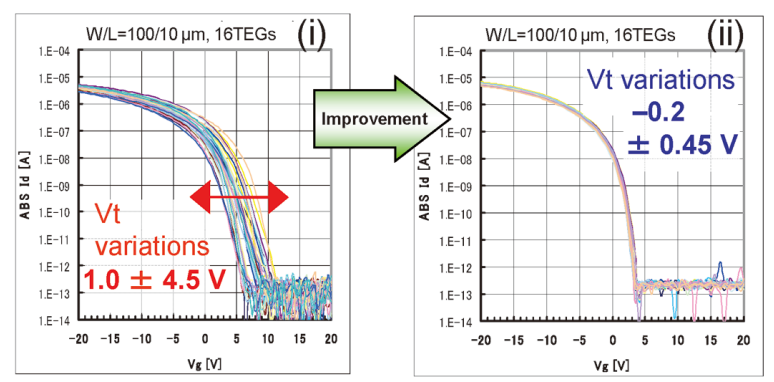

Fig.2 Threshold voltage variations in OTFTs with a polymer GI: (i) without surface treatment on the polymer GI; (ii) with surface treatment.

surface of the polymer GI by rinsing with a variety of solutions. We have previously reported a procedure for cleaning the GI surface, which was to remove the residue from the $\mathrm{SiO}_{2}$ GI surface using $1 \%$ hydrogen fluoride solution ${ }^{7)}$.

For the polymer GI used in this study, however, hydrogen fluoride solution proved unsuitable for use as a cleaning solution, since it had no effect of liftoff from the polymer surface. Instead, we used an alkaline solution to remove the $\mathrm{MoOx}$ residue. Fig.3 illustrates our approach to the surface treatment of the polymer GI. The results, however, showed that it did not completely remove the residue from the polymer GI surface: the $\mathrm{x}$ ray photoelectron spectroscopy (XPS) spectra (i)-(b) in Fig.4 showed that MoOx remained on the polymer GI.

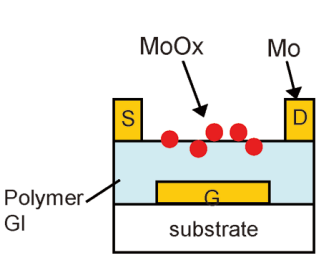

(a)

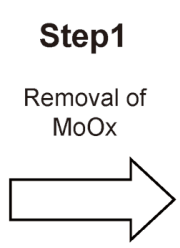

Rinsing with an alkaline solution

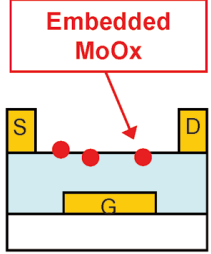

(b)

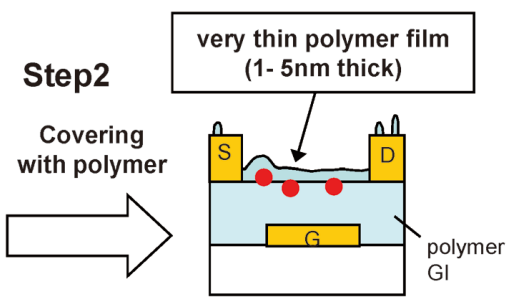

(c)

Fig.3 Polymer GI surface treatment flowchart. Step 1 shows attempted removal of MoOx residue using an alkaline solution, followed (Step 2) by covering the surface with a very thin film of polymer GI material. (a) Schematic cross-sectional view of polymer GI surface condition without treatment after wet etching process of the SD electrode, (b) MoOx residue remaining embedded in the polymer GI after rinsing an alkaline solution and (c) Covering the embedded MoOx residue using very thin polymer GI, also showing aggregated polymer GI material on the SD electrode.

(i)

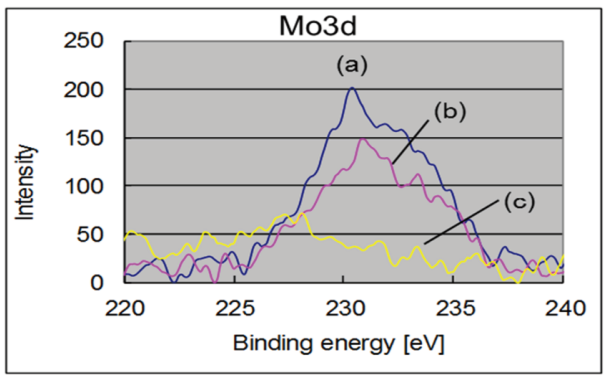

(ii)

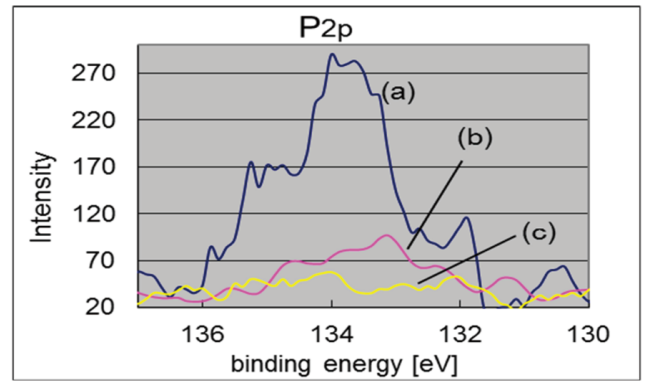

Fig.4 XPS (i) Mo3d and (ii) P2p peaks on the polymer GI after the wet etching process of the SD electrode. (a) without treatment, (b) after rinsing with an alkaline solution and (c) after rinsing and polymer coating. 
In contrast, the XPS data (ii)-(b) in Fig.4 showed that etchant residue (including phosphoric acid) using the SD electrode patterning was reduced on rinsing with an alkaline solution. Our subsequent analysis of the polymer GI surface condition using secondary ion mass spectrometry (SIMS) revealed the Mo residue to be actually embedded in the depth direction of the polymer GI (as shown in Fig.3-(b)). We therefore tested whether a very thin polymer GI would be suitable for covering the embedded $\mathrm{MoOx}$ residue. Fig.3-(c) shows a schematic cross-sectional view of the OTFT covered with very thin polymer GI. The film was approximately 1 - $5 \mathrm{~nm}$ thick. Its morphology on the SD electrode is different from that on the polymer GI due to wettability, observed by using AFM techniques. The very thin polymer on the SD electrode indicated the aggregate condition shown in Fig.3-(c).

The XPS spectra in Fig.4 (i)-(c) also showed that, after the surface treatment, no more residue was present. We concluded that the embedded residue in the surface can be covered by coating with a very thin polymer GI material layer (less than $5 \mathrm{~nm}$ thickness). This results in a drastic reduction in Vt variation of the OTFTs.

The second problem was that when the OSC was fabricated using an inkjet process, the OSC film on the polymer GI was very thin, since the OSC ink was drawn to the bank wall in a kind of coffee-ring effect, resulting in considerable non-uniformity (Fig.6 (ii)-(a)) ${ }^{7)}$. Fig.5 shows our data revealing that the Vt variation in OTFTs (i )

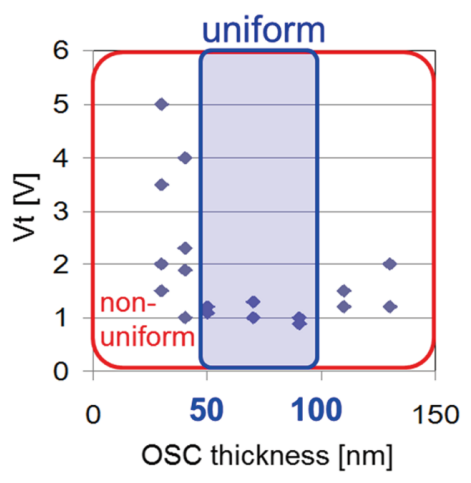

( i i )
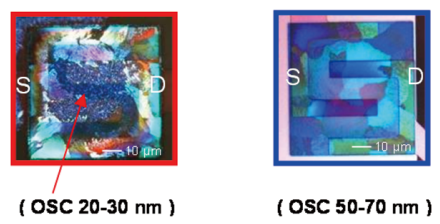

Fig.5 (i) Vt variation in OTFTs vs. OSC film thickness and (ii) Top view of micrographs of OSC film morphology. Area enclosed by blue line has uniform OSC crystallinity (the grain size is $20-40 \mu \mathrm{m}$ ); area enclosed by red line has nonuniform crystallinity (the grain size of the OSC is less than $1 \mu \mathrm{m})$.

depends on OSC film thickness and crystallinity. For example, if an OSC film is thinner than $50 \mathrm{~nm}, \mathrm{Vt}$ variation is high and shifts toward a positive voltage, and the OSC crystallinity is also lower: the grain size of the OSC is less than $1 \mu \mathrm{m}$ (area enclosed by red line in Fig.5-(ii)). Based on these results, we estimated the ideal OSC film thickness to be $50-100 \mathrm{~nm}$. We then tried to control the OSC film's thickness and uniformity.

(a) non-uniform

(b) uniform

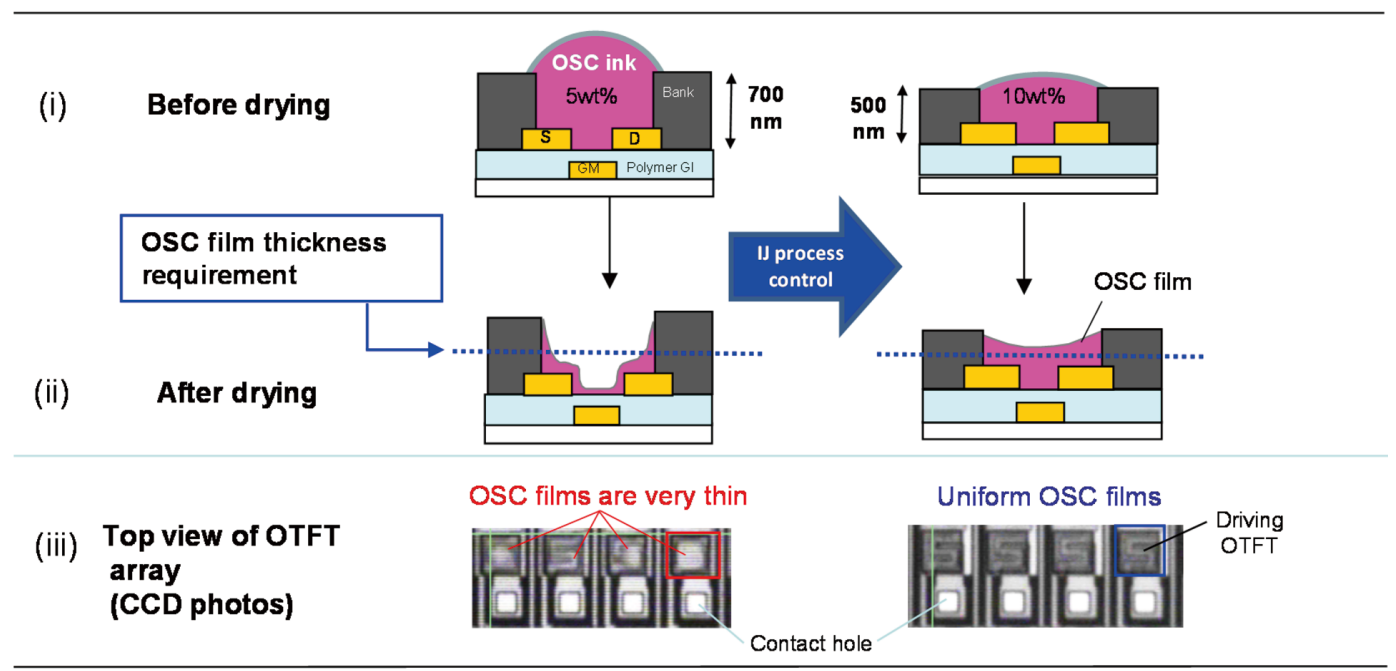

Fig.6 Schematic cross-sectional view of OSC film fabricated using the inkjet process: (i) before OSC ink has dried and then (ii) after drying. The OSC film thickness was controlled by bank height and OSC ink concentration: (a) non-uniform and (b) uniform process conditions. (iii) Top view CCD photographs in-plan of the OTFT array: (iii)-(a) non-uniform and (iii)-(b) uniform in-plan of the OTFT array. 


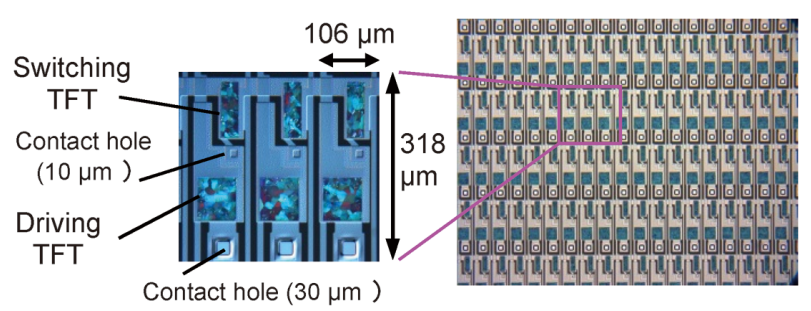

Fig.7 Top view of the 80-ppi OTFT array with a polymer GI, and a sub-pixel area.

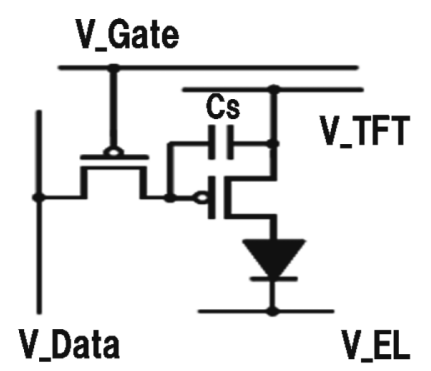

Fig.8 A schematic diagram of the pixel circuit for the flexible AMOLED display with OTFTs.

Fig. 6 shows a schematic cross-sectional view of an OSC film fabricated using the inkjet process. (i) and (ii) show before and after the OSC ink has dried. (iii) comprises CCD photographs of variations in the OTFT array. Fig.6 (iii)-(a) shows non-uniform, that is, white color parts in the photographs comprises, indicating very thin OSC film. To improve the non-uniformity property in Fig.6-(a), our strategy was to change the bank height and the OSC ink concentration. The numerical values are as follows: from $700 \mathrm{~nm}$ to $500 \mathrm{~nm}$ and from $5 \mathrm{wt} \%$ to $10 \mathrm{wt} \%$ respectively.

Both approaches indicate how to quickly adjust the drying speed of the OSC ink, and from the results, we were able to control the OSC film's thickness and uniformity as shown Fig.6-(b).

We therefore achieved a marked reduction in $\mathrm{Vt}$ variation of the OTFTs, with the uniformity of the Vt being $-0.2 \pm 0.45 \mathrm{~V}$ as shown in Fig.2-(ii).

\subsection{OTFT array and pixel circuit}

We fabricated the 80-ppi OTFT array shown in Fig.7 using an inkjet process. The pixel size is $318 \times 318 \mu \mathrm{m}$. Each sub-pixel consists of two transistors and one capacitor (2T1C). The W/L, the ratio of channel width to channel length of the driving and the switching OTFT are $100 / 10 \mu \mathrm{m}$ and $70 / 10 \mu \mathrm{m}$ respectively. Fig.8 shows the pixel circuit used for the flexible AMOLED display.

\subsection{Flexible AMOLED display}

A 4-inch, mono-color OLED display driven by the OTFTs with the polymer GI on a plastic substrate was fabricated as shown in Fig.9. The specifications of the

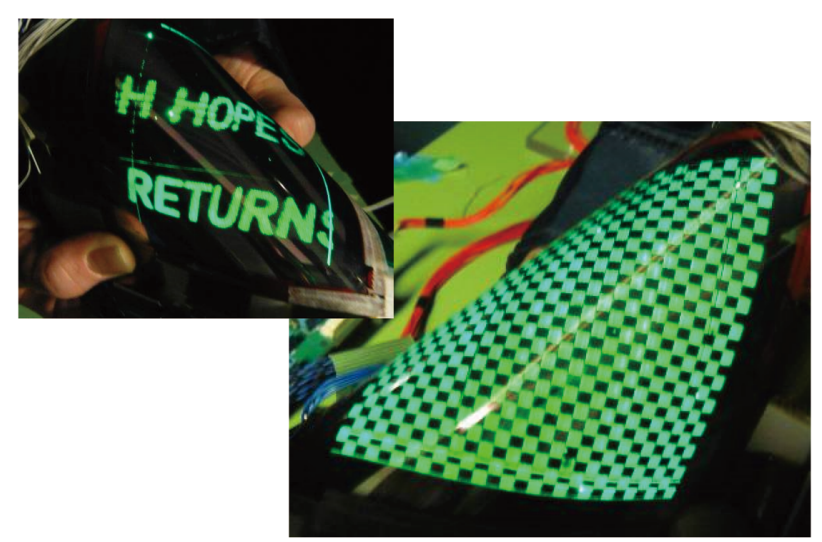

Fig.9 Photographs of 80-ppi OTFT-driven flexible OLED display with moving images while being bent.

Table 1 Specifications of the flexible AMOLED display.

\begin{tabular}{l|l|}
\hline Display size & 4 inch \\
\hline Number of pixels & $224 \times 224$ \\
\hline Pixel size & $318 \mu \mathrm{m} \times 318 \mu \mathrm{m}$ \\
\hline Resolution & $80 \mathrm{ppi}$ \\
\hline Operation Scheme & $2 \mathrm{TFT} 1 \mathrm{capacitor}$ \\
\hline Peak luminance & $>100 \mathrm{~cd} / \mathrm{m}^{2}$ \\
\hline Supply Voltage & $14 \mathrm{~V}$ \\
\hline Gate Voltage & High $15 \mathrm{~V}$ \\
& Low $-10 \mathrm{~V}$
\end{tabular}

(i )

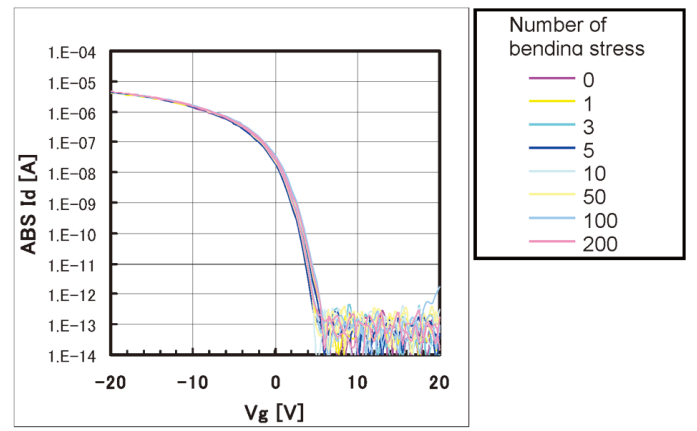

(ii )

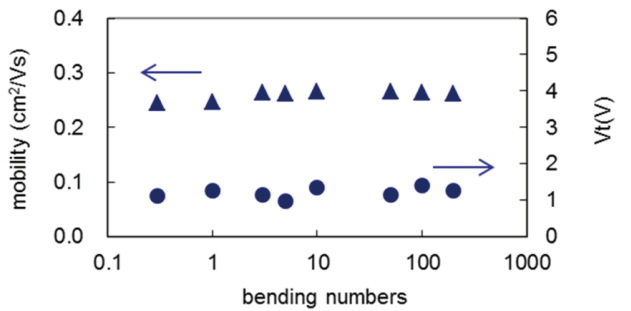

Fig.10 (i) Transfer curves, (ii) mobility and Vt of the OTFT after bending 200 times through a radius of $5 \mathrm{~mm}$.

display are summarized in Table $\mathbf{1}$.

Fig.10-(i) and (ii) show the transfer curves, the mobility and the Vt of the OTFT during bending 200 
times with a radius of curvature of $5 \mathrm{~mm}$. No degradation of the OTFT characteristics was observed after the test. The flexible AMOLED display provided clear moving images, with few bright dots during bending, as shown in Fig.9. The mechanical stability of this display proved to be very high. This is promising technology for future flexible electronic devices.

\section{Conclusions}

We have successfully fabricated a 4-inch 80-ppi flexible AMOLED display driven by OTFTs with a polymer GI on a plastic substrate. The Vt variations in the OTFTs were dramatically suppressed by controlling the surface condition on the polymer GI, from which the residues were removed by cleaning with alkaline solution and re-covered with the same material as the polymer GI, after which the OSC film thickness was controlled by bank height and OSC ink concentration. Consequently the uniformity of the Vt indicated $-0.2 \pm$ $0.45 \mathrm{~V}$, which as a result drastically improved the image quality of the flexible AMOLED display. No degradation of the OTFT characteristics was observed after 200 times bending over a 5 -mm radius.

\section{Acknowledgements}

The authors would like to thank Mitsubishi Chemical Corporation for providing the organic semiconductor and polymer materials (bank, polymer GI, over-coating), and Sumitomo Chemical Co., Ltd. for providing the undercoating material and polymer GI.

\section{[References]}

1) M. Katsuhara, I. Yagi, A. Yumoto, M. Noda, N. Hirai, R. Yasuda, T. Moriwaki, S. Ushikura, A. Imaoka, T. Urabe and K. Nomoto, "A flexible OLED display with an OTFT backplane made by scalable manufacturing process," Journal of the SID 18/6, 399 (2010)

2) Y. Nakajima, Y. Fujisaki, T. Takei, H. Sato, M. Nakata, M. Suzuki, H. Fukagawa, G. Motomura, T. Shimizu, Y. Isogai, K. Sugitani, T. Katoh, S. Tokito, T Yamamoto and H. Fujikake, "Low-temperature fabrication of 5-in. QVGA flexible AMOLED display driven by OTFTs using olefin polymer as the gate insulator," Journal of the SID, 19/12, 861 (2011)

3) Makoto Noda, Norihito Kobayashi, Mao Katsuhara, Akira Yumoto, Shinichi Ushikura, Ryouichi Yasuda, Nobukazu Hirai, Gen Yukawa, Iwao Yagi, Kazumasa Nomoto and Tetsuo Urabe, "An OTFT-driven rollable OLED display," Journal of the SID 19/4, 316 (2011)

4) Y. Nakajima, T. Takei, Y. Fujisaki, H. Fukagawa, M. Suzuki, G. Motomura, H. Sato, T. Yamamoto and S. Tokito, "Improvement in image quality of a 5.8-in. OTFT-driven flexible AMOLED display," Journal of the SID, 19/1, 94 (2011)

5) C. Harada, S. Ohta, T. Chuman, H. Ochi, H. Sato, S. Ishizuka and A. Yoshizawa, "Organic Thin Film Transistors with Ink-jetted Organic Semiconductor Layer and 3-inch QQVGA AM-OLED Panel," IDW '07, 1977 (2007)
6) C. Harada, T. Hata, T. Chuman, S. Ishizuka and A. Yoshizawa, "Solution-Processed Organic Thin-Film Transistor Array for ActiveMatrix Organic Light-Emitting Diode," Jpn.J. Appl. Phys. 52, 05DC22 (2013)

7) Y. Okumoto, T. Ukeda, M. Matsui, K. Okumoto, A. Miyamoto and K. Morita, "Top-emission AMOLED display driven by organic TFTs with semiconductor layer patterned by inkjet process," Journal of the SID, 20/10, 575 (2012)

8) M. McDowell, I.G. Hill, J.E. McDermott, S.L. Bernasek and J. Schwartz, "Improved organic thin-film transistor performance using novel self-assemble monolayers," Appl. Phys. Lett. 88, 073505 (2006)

9) K.P. Pernstich, S. Haas, D. Oberhoff, C. Goldmann, D.J. Gundlach, B. Batlogg, A.N. Rashid and G. Schitter, "Threshold voltage shift in organic field effect transistors by dipole monolayers on the gate insulator," J. Appl. Phys. 96, 6431-6438 (2004)

10) S. Kobayashi, T. Nishikawa, T. Takenobu, S. Mori, T. Shimoda, T. Mitani, H. Shimotani, N. Yoshimoto, S. Ogawa and Y. Iwasa, "Control of carrier density by self-assembled monolayers in organic field-effect transistors," Nat. Mater. 3, 317-322 (2004)

11) K. Suemori, S. Uemura, M. Yoshida, S. Hoshino, N. Takada, T. Kodzasa and T. Kamata, "Threshold voltage stability of organic field-effect transistors for various chemical species in the insulator surface," Appl. Phys. Lett. 91, 192112 (2007)

12) S.A. DiBenedetto, A. Facchetti, M.A. Ratner and T.J. Marks, "Molecular Self-Assembled Monolayers and Multilayers for Organic and Unconventional Inorganic Thin-Film Transistor Applications," Adv. Mater. 21, 1407-1433 (2009)

13) L.L. Chua, J. Zaumseil, J.F. Chang, E.C.W. Ou, P.K.H. Ho, H. Sirringhaus and R.H. Friend, "General observation of n-type fieldeffect behaviour in organic semiconductors," Nature 434, 194-199 (2005)

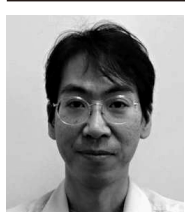

Akihito Miyamoto joined Panasonic Corp. in 1992, where he engaged in research on organic semiconductor devices. At present, he is in charge of printable TFT at the R\&D Division. He is a member of JSAP, M\&BE and JPS

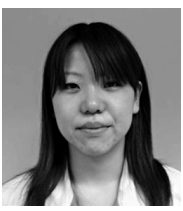

Yuko Okumoto received B. Eng., M. Eng. Degrees from Osaka University in 2005, 2007 respectively. In 2007, she joined Panasonic Corp. Since then, she has been engaged in the research and development of organic TFT at the R\&D Division.

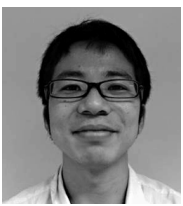

Kenichi Sasai received BEng and MEng degrees from Osaka University in 2007 and 2009 respectively. In 2009, he joined Panasonic Corp. Since then, he has been engaged in the research and development of organic TFT at the R\&D Division.

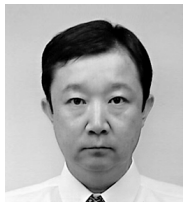

Kiyoyuki Morita received B. Eng. and M. Eng. Degrees from Osaka University in 1983 and 1985, respectively. In 1985, he joined Matsushita Electric Industrial Co., Ltd., (presently Panasonic) where he engaged in research on silicon ULSI process and devices, quantum effect devices, and organic electronics. He received the degree of $\mathrm{PhD}$. from Osaka University. At present, Dr. Morita is in charge of research on flexible displays as a general manager of Device Solution Center. He is a member of JSAP and SCEJ. 